\title{
Commentary by Pedro A. Morettin
}

This volume honors the work of David Brillinger in several areas, but most notably in the fields of point processes and of time series analysis and applications. It has been a privilege to me to have been his $\mathrm{PhD}$ student at Berkeley and then become a friend for the past 40 years. It has been quite a journey. David's work on time series has been influential to many people, especially for his students(over forty), many of them who pursued careers in time series and related fields. He is well know in Brazil, for his constant visits and valuable collaboration over the years. His book "Time Series: Data Analysis and Theory" became a classic and I was very fortunate to attend his classes using an earlier draft of the book.

In his assessment of the works of John W. Tukey, David wrote: "Anyone who has been involved with John has indeed been fortunate. They have probably remarked upon his rapid domination of the situation at hand, his extensive knowledge of pertinent physical background, his leaps in unthought of directions to concrete procedures, his vocabulary and possibly even his humor." I could not write anything more perfect that applies to David himself. His works pervaded several areas and disciplines, and some of them will be discussed in this volume.

I will concentrate on some articles in time series analysis, starting from some seminal works on second order spectra and polyspectra. Some of the papers are the kind of work that David likes to write on: they bring examples illustrating the interplay between the theory of time series, point processes, spatial processes and areas of applications, like geophysics, neurophysiology, sports, physics etc. I had read most of the papers, but reading them again was a wonderful experience and I hope this will be the case for the readers of this book.

\section{An Introduction to polyspectra [1965]}

I begin with this paper, because I think it is one of the most influential papers written by David. The purpose of the work is to derive: a) certain 
mathematical properties of polyspectra; b) estimates of polyspectra based on an observed stretch of a time series; c) certain statistical properties of proposed estimates and d) several applications of the results obtained.

The term polyspectrum is due to John Tukey. Polyspectra generalizes spectrum and bispectrum for a single time series and cross-spectra for a pair of time series. The author discusses why to use cumulants and not moments in the definition of polyspectra.

A particular class of discrete or continuous $k$-dimensional complex-valued processes is defined, and for members of this class the polyspectrum is defined as the Fourier transform of a cumulant of some order, assumed to exist. Some estimation procedures are considered (e. g. moment-type estimators and estimators based on complex demodulation) and asymptotic (complex) normal distributions are derived for the estimators.

Curiously enough the paper concludes with a note of pessimism on the use of polyspectra.

\section{Asymptotic Theory of Estimates of $k$-th order Spectra [1967]}

This work considers a vector of strictly stationary processes, all moments existing. Under mixing conditions given in terms of cumulants, the $k$-th order cumulant spectral density is defined as the Fourier transform of the corresponding $k$-th order cumulant of the process. Estimates for the cumulant spectral densities are provided and their properties derived. The proposed estimates are weighted averages of periodograms and asymptotic unbiasedness, joint normality and covariance structure are obtained. Some remarks on aliasing and previous works are made.

\section{Asymptotic Properties of Spectral Estimates of Second Order [1969]}

This article considers an $r$-variate strictly stationary, zero mean, stochastic process, satisfying some mixing condition. From the finite Fourier transform of $T$ observations of the process, the periodogram and other estimates are proposed, namely estimates of the spectral measure, the autocovariance function and spectral density.

The asymptotic unbiasedness and asymptotic distributions of the estimates are derived. Under additional conditions, the asymptotic distribution of the periodogram is complex Wishart, for the spectral measure estimate is multivariate normal, the same for the autocovariance estimates and spectral density estimates. Some departures from the assumptions are commented on. 


\section{Fourier Analysis of Stationary Processes [1974]}

This is an invited review paper on Fourier analysis (FA) of stationary processes written for the Proceedings of the IEEE. It begins with a description of important procedures in FA, including the estimation of the spectrum, fitting of parametric models and identification of linear systems.

The topics surveyed include: stationary real-valued discrete time series, the finite Fourier transform, the estimation of the spectrum, parametric models, linear models, vector-valued continuous spatial series, stationary point processes and stationary random Schwartz distributions.

Enphasis is on the large sample properties of estimators. Final remarks are made on higher order spectra and nonlinear systems.

\section{The Digital Rainbow: Some History and Applications of Numeri- cal Spectrum Analysis [1993]}

This paper focus on the spectrum of a phenomenon. This is viewed as a display of the intensity of the phenomenon versus frequency. Some historical development of the field of spectrum analysis is given, with contributions of Michelson (1892), Schuster (1898), Einstein (1914), Fisher (1929), Bartlet (1950), Tukey (1958) and Yaglom (1987).

Some applications are given on: a) the free oscillations of the Earth, with an example of the 1960 Chilean earthquake; b) seismic surface waves (earthquake waves whose energy is trapped near the Earth's surface) and c) nuclear magnetic resonance (NMR) spectroscopy.

The paper concludes with some discussion on future prospects.

\section{An Investigation of the Second-and Higher-order Spectra of Music [1998]}

In this work the authors describe the two basic representations of music (signal and score representations), review some previous investigations and then present results of modelling second and higher order spectra in order to assess Gaussianity and linearity. They also discuss time series and marked point process representations. Four models for the spectra are considered (one of them being the $1 / f$ noise model) and these are fitted to some scores, in particular the Bach's Coffee Cantata. 'Another feature is the fit of $1 / f$ model to 12 music scores, ranging from Baroque to Latin music, concluding that it fits well. 


\section{Some Examples of Empirical Fourier Analysis in Scientific Prob- lems [1999]}

This is another paper describing some interesting applications of Fourier analysis in real problems. The article starts by stating the importance of Fourier analysis and them reviews some physical examples of the methodology. It continues giving some analytical background on Fourier and wavelet analysis and moves to stationary processes, central limit theorems and shrinking. Finally gives four examples, in electron microscopy, seismic surface waves, nuclear magnetic resonance spectroscopy and a wavelet analysis of microtubule movement (linear polymers basic to cell motility). The paper is concluded with some open problems.

\section{Some Examples of Random Process Environmental Data Analysis [2000]}

This paper presents examples in the environmental sciences. The processes analyzed range from point and marked point processes, to time series, spatial-temporal processes and particle processes. Preliminary some basic concepts and methods on random processes and inference are set down.

For the point process case an example from space science is given. The data consists of counts of orbiting debris, that may cause problems for space crafts. Statistics as the average periodogram, autointensity and coherence estimates are employed. Interest lie in the type of point process that better represent the data, the size of the particles (marks) and questions of independence of marks and times, and of altitudes and sizes.

In the case of a time series, the example is from Public Health. The data for analysis consists of average number of daily births in Toronto in 1986 . The interest is in high level of cesarean deliveries. The counts are modelled by a Poisson with trending mean.

For spatio-temporal processes it is given an example from Neuroscience, concerning the olfactory system, data collected of the response of rabbits sniffing an odor. A random effects model is proposed followed by Fourier transformation.

Finally, for particle processes (path or trajectory of an object moving along a line), an example from Ecology is given, namely the migration path of an elephant seal. The proposed model is a nonlinear state space model.

The paper concludes with a discussion of other types of processes, data and techniques. 